\title{
Multiple-charged optical vortex solitons: existence and stability in saturable Kerr-type nonlinear media
}

\author{
A. Dreischuh", G. G. Paulus ${ }^{\mathrm{b}}$, D. Neshev ${ }^{\mathrm{a}}$, F. Grasbon ${ }^{\mathrm{b}}$, H. Walther ${ }^{\mathrm{b}, \mathrm{c}}$ \\ ${ }^{a}$ Sofia University, Department of Quantum Electronics, 5, J. Bourchier Blvd., BG-1164 Sofia, Bulgaria \\ bMax-Planck-Institut für Quantenoptik, Hans-Kopfermann-Str. 1, D-85748 Garching, Germany \\ ${ }^{\mathrm{c}}$ Ludwig-Maximillians-Universität, Sektion Physik, Am Coulombwall 1, D-85747 Garching, Germany
}

\begin{abstract}
Multiple-charged optical vortex solitons (OVSs) are generated in a thermal nonlinear medium with saturation. The respective soliton constants are found to be linearly proportional to the topological charges. The linear stability analysis and the numerical simulations indicate a rich variety of instability scenaria depending on the type of perturbation. The saturation of the nonlinearity is shown to be able to slow down the decay of multiple charged dark beams at an intermediate evolution stage and to prevent their ultimate decay into charge-one OVSs. This concept is experimentally verified by the experimental observation of a partial decay of a triple-charged optical vortex beam.
\end{abstract}

Keywords: phase dislocation, topological charge, nonlinear medium, saturation, vortex soliton, stability

\section{INTRODUCTION}

The only known truly two-dimensional dark spatial solitons (DSSs) are the optical vortex solitons (OVSs) ${ }^{1}$. They are characterized as localized self-supporting intensity dips with screw phase dislocations imposed on bright background beams. In self-defocusing nonlinear media (NLM) such solitons are generated as a result of the balance between diffraction and nonlinearity ${ }^{1}$. The characteristic helical phase profile (Fig. 1a) is analytically described by an $\exp \{\operatorname{im} \varphi$ \} multiplier, where $\varphi$ is the azimuthal coordinate and the integer number $m$ is the so called topological charge (TC). As compared to the single-charged OVSs, these with TCs $|m|>1$ are less investigated. A vortex of charge $|m| \geq 2$ is found to be topologically unstable against bifurcation into $|m|$ branches ${ }^{2}$. The nonlinear Schrödinger equation (NLSE), which describes the OVS formation, can be considered as a special kind of the Ginzburg-Landau equation, for which multiple-charged vortices may be stable in a certain range of parameters ${ }^{3}$. The saturation of the third-order nonlinearity involved in the generalized NLSE is proven to suppress effectively the DSS transverse instability ${ }^{4}$. The later motivated this experimental study ${ }^{5}$ of the generation and stability of OVSs with topological charges $m=2 \ldots 4$ in a saturable self-defocusing nonlinear medium.

\section{EXPERIMENTAL RESULTS}

The first step in this experiment was to evaluate the quality of the OV beams used. These are created by illuminating photolitographically fabricated computer-generated holograms (CGHs) with an $\mathrm{Ar}^{+}$-ion laser beam ( $\lambda=488 \mathrm{~nm}$ ). The binary type CGHs are produced on a common substrate at a grating period of $20 \mu \mathrm{m}$. Within the CGHs it is easy to recognize the center of the $m$-fold charged vortex beam by the converging of $m$ neighboring lines in one (Fig. 1b). Making use of a Mach-Zehnder interferometer and the four-frame technique for interferogram analysis ${ }^{6}$ we reconstructed the phase distributions of the OV beams. From the retrieved diametrical phase distribution of a single-charged OV (Fig. 2) we estimated that the deviation of the phase jump from the required value of $\pi$ is within $\pi / 20$. This value is in reasonable agreement with the unavoidable quantization inaccuracy of $\pi / 24$ for binary CGHs. The ratio of the background beam radius $r_{\mathrm{BG}}$ to the $\mathrm{OV}$ beam radius $\mathrm{r}_{\mathrm{OV}}$ (both measured as half-widths at half-maxima at the entrance of the nonlinear medium) is $\mathrm{r}_{\mathrm{BG}} / \mathrm{r}_{\mathrm{OV}}=20,14,12$, and 11 for $|m|=1,2,3$, and 4, respectively. Although the host beam can not be considered to be an infinite one, it is wide enough to prevent the vortex-to-background interaction. 


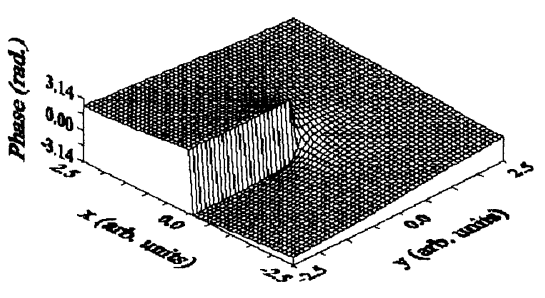

(a)

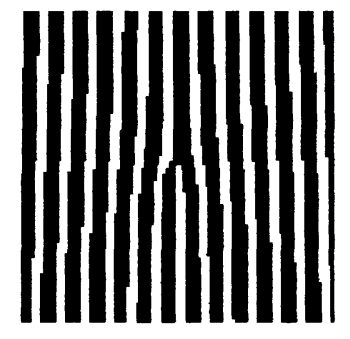

(b)

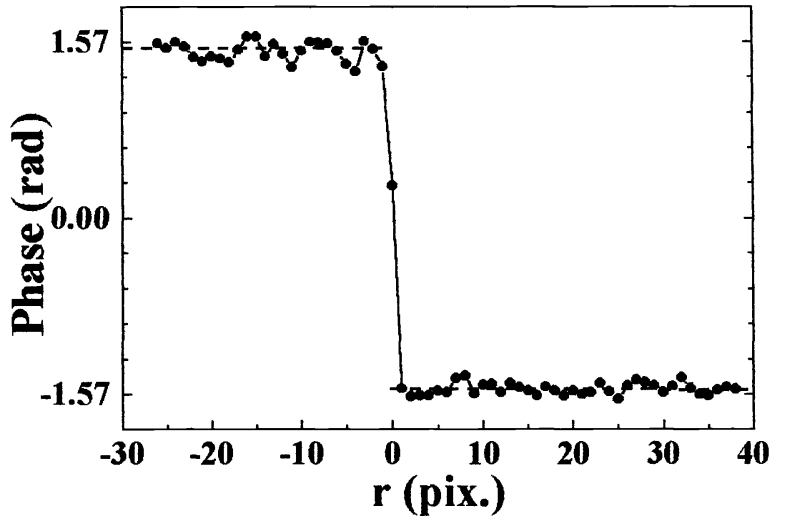

Fig. 2 Diametrical phase distribution of a single-charged OV beam extracted from the experimental data. Dashed line mean values of the phase outside the vortex core.

The experimental setup for investigating the OVS formation is shown in Fig. 3. The first-order background beam with the OV nested in is transmitted through a slit placed behind the CGH and is subsequently focused on the entrance of the NLM (ethylene glycol dyed with DODCI to reach an absorption of $0.107 \mathrm{~cm}^{-1}$ at $\lambda=488 \mathrm{~nm}$ ). After passing the desired nonlinear propagation path-length the dark beam is partially reflected by a prism that is immersed in the liquid to project the beam directly on a CCD-array. The immersed prism, one filter set and one of the cameras are mounted on a translation stage enabling us to scan nonlinear propagation path-lengths ranging from $0.5 \mathrm{~cm}$ to $8.5 \mathrm{~cm}$. The second filter set is placed on a rotation stage and provides the possibility to record unsaturated interference pictures after the maximal NLM length of 10 $\mathrm{cm}$. The same camera is also utilized to monitor the far-field energy-density distribution and to identify the development of a modulational instability.

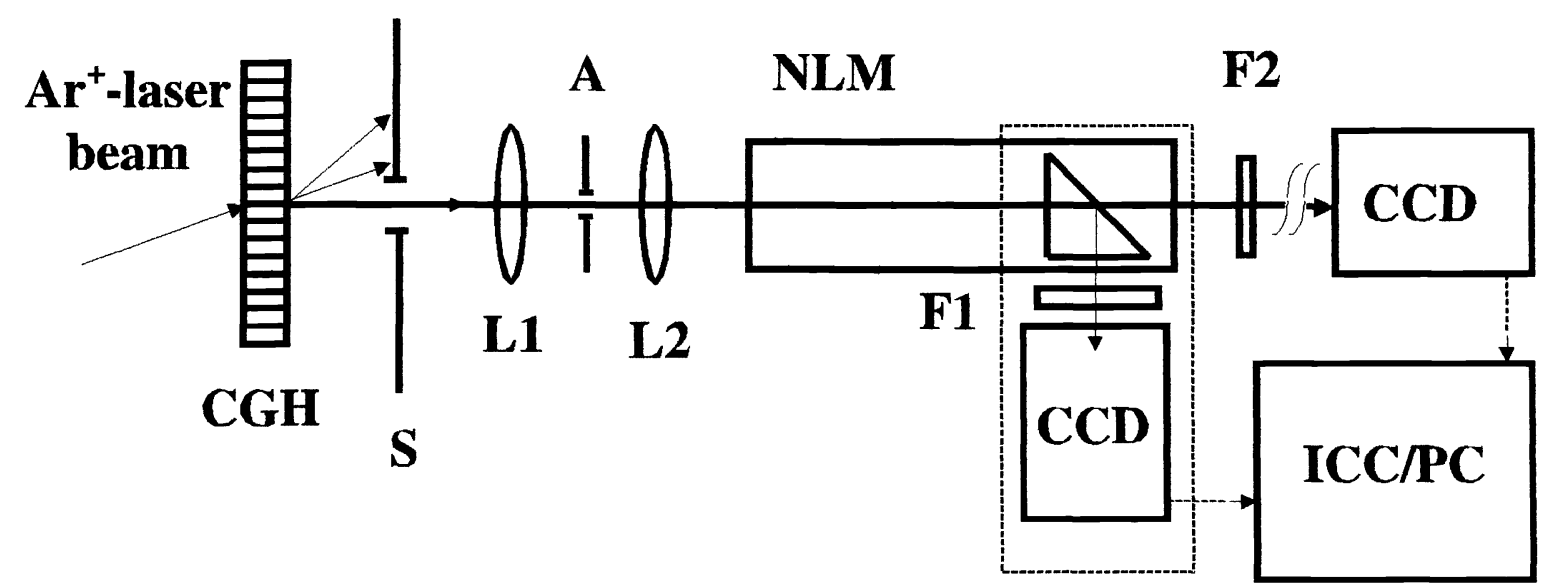

Fig. 3 Experimental arrangement used to study the evolution of multiple-charged OVSs along the thermal nonlinear medium.

(CGH - computer-generated hologram; S - slit; L1, L2 - AR-coated lenses ( $\mathrm{f}=80 \mathrm{~mm}$ ); A- iris aperture; F1,F2 - filter sets; CCD -

charge-coupled device camera of $13 \mu \mathrm{m}$ resolution; PC/ICC - personal computer equipped with an image capturing card).

In Fig. 4 we present experimental data for the dependence of the quantity $\mathrm{Ia}^{2}$ (the product of the background beam intensity and the square of the dark beam radius) for an OV beams with topological charges $|m|=1$ (a), 2 (b), 3 (c), and 4 (d), respectively. Since multiple-charged vortex beams are expected to be unstable against decay into single-charged ones, we tried carefully to minimize the aberrations introduced by the focusing system. Asymptotic stabilization of the quantity $\mathrm{Ia}^{2}$ with increasing background-beam power means that the respective soliton constant is reached. The pronounced deviation of the last three points in Figs. $4 \mathrm{~b}$ allows us to state that we are able to distinguish clearly between stable double- 
charged OVSs and single-charged OV beams with overlapping cores (triangles). Complete separation between the vortices in the far field is not observed.

By keeping the background beam power/intensity unchanged and translating the prism immersed in the NLM we obtained the dependencies of the $\mathrm{OV}$ beam $\mathrm{r}_{\mathrm{OV}}$ on the nonlinear propagation path-length $z$ for different TCs. Our starting hypothesis ${ }^{5}$ (well motivated by Figs. 4a-d) is that the soliton constant of a $m$-fold charged OVS equals $|m|$ times the soliton constant of a single-charged OVS.
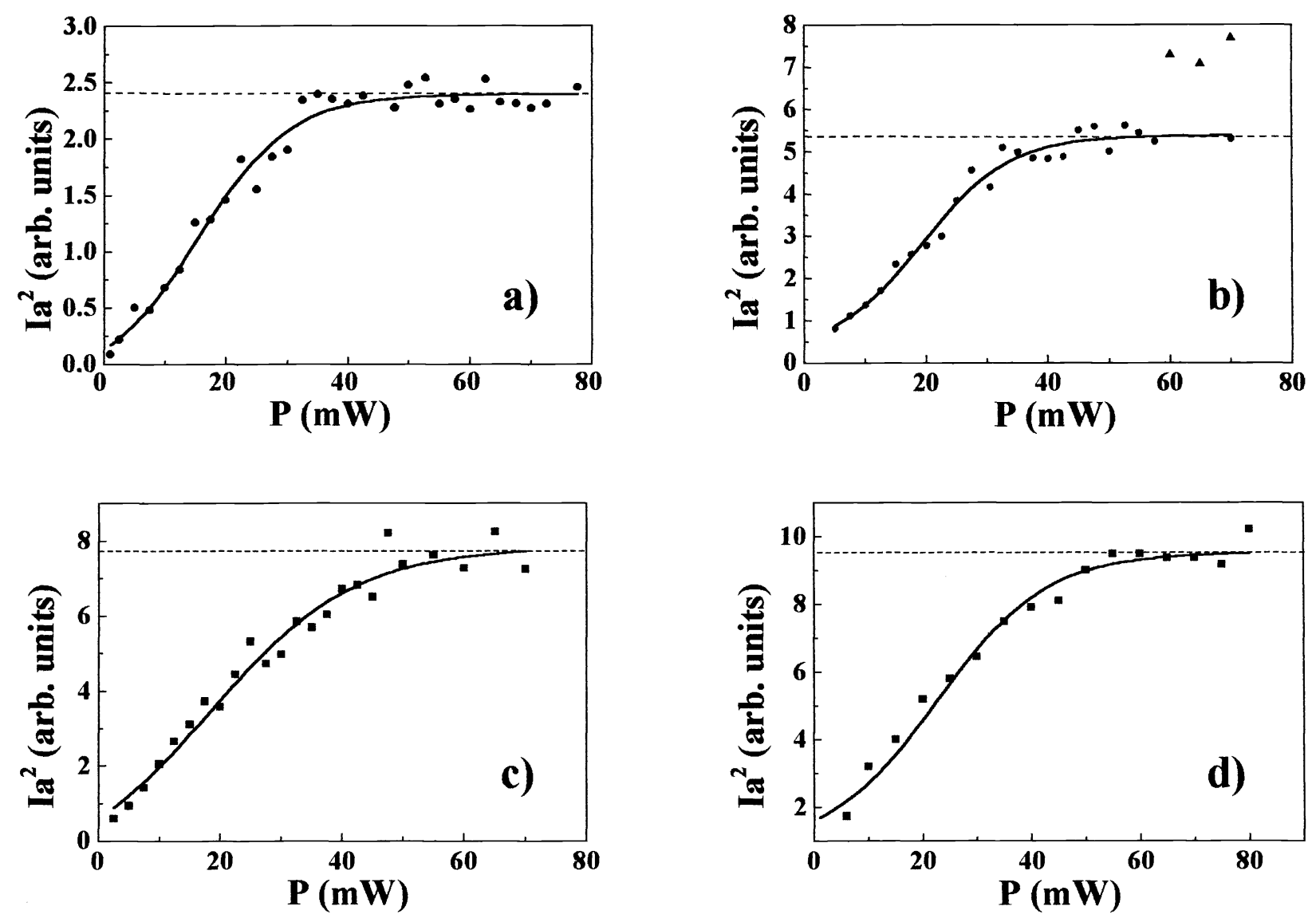

Fig. 4 Power dependence of the quantity $\mathrm{Ia}^{2}$ for an OVSs of topological charges $|m|=1(\mathrm{a} ; \mathrm{z}=8 \mathrm{~cm}), 2(\mathrm{~b} ; \mathrm{z}=7 \mathrm{~cm}), 3(\mathrm{c} ; \mathrm{z}=6 \mathrm{~cm})$, and 4 $(\mathrm{d} ; \mathrm{z}=5.5 \mathrm{~cm})$ demonstrating saturation and stabilization. (solid curves - best fits; dashed lines - the respective soliton-constant values).

Accounting for the focusing conditions (i.e. for the Rayleigh diffraction length $L_{D i f f}$ of the vortex core) and for the absorption in the NLM we calculated reverse the topological charges $m$ '. The data summarized in Table 1 show the perfect agreement between the TCs encoded in the holograms and those recalculated for the respective OVSs. This provides a strong evidence for the linear proportionality of the soliton constants to the charges.

Encoded in the CGHs and recalculated topological charges

\begin{tabular}{ccc}
\hline$m$ & $\mathrm{~L}_{\text {Diff }}(\mathrm{cm})$ & $m^{\prime}$ \\
\hline 1 & 2.5 & $1( \pm 0.05)$ \\
2 & 3.2 & $2( \pm 0.08)$ \\
3 & 3.2 & $3( \pm 0.05)$ \\
4 & 3.1 & $4( \pm 0.01)$ \\
\hline
\end{tabular}




\section{STABILITY ANALYSIS}

The propagation of (2+1)-dimensional continuous-wave optical beams in a nonlinear medium is described by the NLSE

$$
-i 2 k_{0} n_{0} \frac{\partial E}{\partial z}+\left(\frac{\partial}{\partial x^{2}}+\frac{\partial}{\partial y^{2}}\right) E+k_{0}^{2} f\left(|E|^{2}\right) E=0,
$$

where $k_{0}$ is the vacuum wavenumber and $n_{0}$ is the linear refractive index of the medium. $E(\mathrm{x}, \mathrm{y}, \mathrm{z})$ is the slowly-varying complex electric field amplitude, and $f\left(|E|^{2}\right)$ describes in a general form the nonlinear contribution to the refractive index

$$
n^{2}\left(x, y,|E|^{2}\right)=n_{0}^{2}(x, y)+f\left(|E|^{2}\right) .
$$

Accounting explicitly for the topological charge $m$ of the OV beam $\left(E(r, z)=U(r, z) e^{i m \varphi}\right)$ and looking for a smallamplitude solution of the NLSE in the form

$$
U(r, z)=\left[U_{0}(r, z)+a(r, z)\right] \exp \{i(\omega r-k(r, z) z+\Phi(r, z))\},
$$

$\left(a^{2}<<U_{0}^{2}\right.$ and $\Phi(r, z)$ are the unknown perturbation amplitude and phase), we obtain an expression for the critical spatial frequency of the perturbation

$$
\Omega_{c r i t}^{2}=\frac{\left[1 / r+2 U_{0}^{2} g^{\prime}\left(U_{0}^{2}\right)\right]\left[\omega^{2}+m^{2} / r^{2}\right]}{k+g\left(U_{0}^{2}\right)}=\frac{\left[1 / r-\left(k_{0} / n_{0}\right) U_{0}^{2} f^{\prime}\left(U_{0}^{2}\right)\right]\left[\omega^{2}+m^{2} / r^{2}\right]}{\left(k_{0} / 2 n_{0}\right)\left[1-f\left(U_{0}^{2}\right)\right]} .
$$

In a saturable NLM $\mid f^{\prime}\left(U_{0}^{2}\right)$ and $\Omega_{c r i t}$ are decreasing functions of the intensity. This confirms the general conclusion that the saturation of the third-order nonlinearity is able to suppress effectively the modulational instability. The increase of the TC $m$ leads to higher values of the critical transverse spatial frequency of the perturbation $\Omega_{c r i t}$ and, therefore, to an enhanced modulational sensitivity of the OVSs. A perturbation with a transverse spatial frequency $\Omega$ satisfying the relation $\left.\Omega_{c r i t}^{2}\right|_{m}>\Omega^{2}>\left.\Omega_{c r i t}^{2}\right|_{m-1}$ is able to initiate a partial decay of triple and higher charged OV beams. Intentional perturbations are caused by horizontal misalignment of the lens $\mathrm{L}_{2}$ (see Fig. 3). The reproducibility of this 'perturbation' is important for the comparison between the decay stages for the OV beams with different topological charges.

The pictures in Fig. 5 are recorded at a nonlinear propagation path length of $6 \mathrm{~cm}$, at a beam power of 72.5 $\mathrm{mW}$, and at the same (for the different TCs) offsets of the focusing lens with respect to its central alignment. The coexistence of displaced phase dislocations with TCs $|m|=2$ and $|m|=1$ as a result of an intentionally caused decay of a triple-charged OV beam is clearly seen (the frame in the dashed circle). A similar picture is recorded at equal but opposite misalignment of the focusing lens which indicates reproducibility. In view of the theoretical model presented in the previous section, these pictures can be explained by the saturation of the nonlinearity, which suppresses effectively the DSS instability.

Separate measurement is done in order to estimate quantitatively the saturation intensity $\mathrm{I}_{\mathrm{sat}}=\mathrm{U}_{0, \text { sat }}^{2}$. The laser beam (without an OV nested in) is cut in half by a knifeedge and is then imaged near the entrance window of the cell. The near-field nonlinear beam self-deflection is measured by direct illumination of the CCD-camera located $1.5 \mathrm{~cm}$ behind the NLM. The best sigmoidal fit yields a saturation power $P_{s a t}=27 \mathrm{~mW}$, This value is weakly sensitive to the particular saturation model.



Fig. 5 Experimentally recorded decay stages for OV beams with different TCs. Dashed circle: partial decay of triple-charged $\mathrm{OV}$ beam at a perturbation causing complete decay of a chargefour beam. 


\section{NUMERICAL SIMULATIONS}

The analysis presented in the previous section is based on linearized equations for the evolution of radial perturbations to the amplitude and phase of the soliton. The perturbations also can depend on the azimuthal angle $\varphi$. Unfortunately, such an ansatz and the subsequent linearization result in partial differential equations. The necessity to study later evolution stages of the vortices and the fact that each initial pure phase (or amplitude) perturbation develops inevitably both amplitude and phase modulation motivated us to carry out extended numerical simulations based on the NLSE. In the case of a pure azimuthal perturbation we obtained the results shown in Fig. 6.

The region of azimuthal instability for OVSs with TCs $m=2$

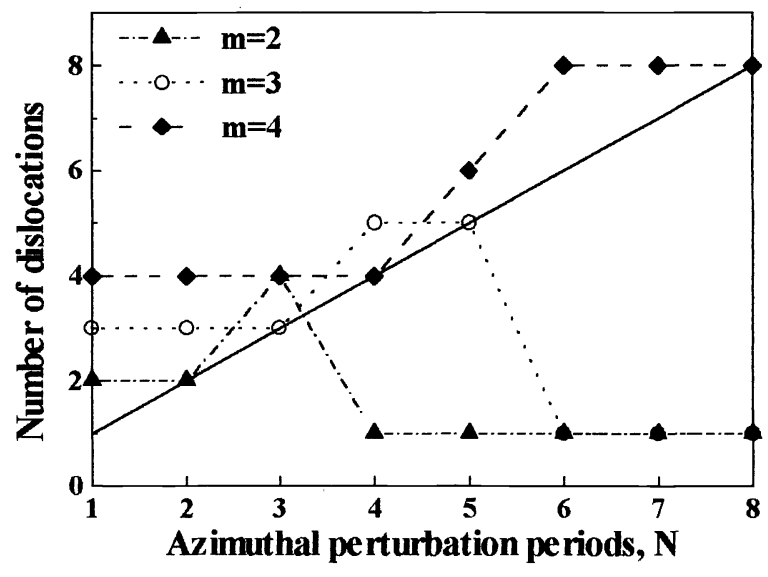

Fig. 6 Number of the screw phase dislocations observed at five nonlinear lengths for azimuthal perturbations of wavenumbers $\mathrm{N}$. (Solid line $-\mathrm{N}=\boldsymbol{m}$ ). and 3 was found to terminate at azimuthal wavenumbers of the perturbations $\mathrm{N}=4$ and 6 , respectively. No upper instability limit vs. $\mathrm{N}$ was reached for the fourfold-charged OVSs at the highest value of $\mathrm{N}_{\max }=8$ in the simulations. (This limit is posed by the fidelity in recognizing clearly the screw phase dislocations at remaining highly overlapped OV-beam cores). The straight line $m=\mathrm{N}$ in Fig.6 is intended to accentuate on the three most important features: At azimuthal wavenumbers $\mathrm{N}$ less than or equal to the TC $m$ the OVSs decay topologically into $m$ vortices with unit circulations. At $\mathrm{N}=m+1$ new pairs of phase dislocations are born. The higher the TC, the larger the number of the vortices. This is a strong indication for the increase of the OVS modulational sensitivity at higher TCs. The transition from topological decay (and creation of new dislocations) to topological (meta)stability at increased $\mathrm{N}$ seems abrupt thus indicating a maximum of the instability growth-rate at $\mathrm{N}>m+1$.

\section{CONCLUSION}

Evidences for the existence of multiple-charged OVSs modulationaly stabilized by saturation of the nonlinearity are presented. The respective soliton constants are found to be linearly proportional to the topological charges. The saturation of the nonlinearity is not known to be able to remove the topological instability, however it reduces the respective instability growth rates. A general expression for the critical transverse spatial frequency for arbitrary charged OVSs imposed to a radial perturbation in a saturable local nonlinear media is derived. The numerical simulations based on the NLSE showed rich variety of instability-evolution scenaria depending on the type of perturbation (azimuthal, radial, or mixed).

\section{ACKNOWLEDGMENTS}

This work was supported by the Science Foundation of the Sofia University (Sofia, Bulgaria). A.D. would like to thank the Alexander von Humboldt Foundation for the award of a fellowship.

\section{REFERENCES}

1. G. A. Swartzlander, Jr., C. T. Law, "Optical vortex solitons observed in Kerr nonlinear media," Phys. Rev. Lett. 69, pp. 2503-2506,1992.

2. $\quad$ J. C. Neu, "Vortices in complex scalar fields," Physica D43, pp. 385-406, 1990.

3. I. S. Aranson, K. A. Gorshkov, A. S. Lomov, and M. I. Rabinovich, "Stable particle-like solutions of multidimensional nonlinear fields," Physica D43, pp. 435-453, 1990.

4. B. Luther-Davies, J. Christou, V. Tikhonenko, and Yu. S. Kivshar, "Optical vortex solitons: experiment versus theory,“J. Opt. Soc. Am. B14, pp. 3045-3053, 1997.

5. I. Velchev, A. Dreischuh, and S. Dinev, "Multiple-charged optical vortex solitons in bulk Kerr media," Opt. Commun. 140, pp. 77-82, 1997.

6. Th. Kreis, Holographic interferometry: Principles and methods, pp. 123-139, Academie-Verlag, Berlin, 1996.

7. D. Rozas, C. T. Law, and G. A. Swartzlander, Jr., „Propagation dynamics of optical vortices, “J. Opt. Soc. Am. B14, pp. 3054-3065, 1997. 